\title{
Multicenter Survey of the Isometric Lower Extremity Strength in Patients with Type 2 Diabetes (MUSCLE-std): Design and Study Protocol
}

\author{
Takuo Nomura ${ }^{*}$, Tomoyasu Ishiguro ${ }^{2}$, Masayoshi Ohira3 ${ }^{3}$ Yukio Ikeda ${ }^{4}$, \\ Masahito Watanabe ${ }^{1}$ \\ ${ }^{1}$ Department of Rehabilitation Sciences, Kansai University of Welfare Sciences, Osaka, Japan \\ ${ }^{2}$ Department of Physical Therapy, Health Science University, Yamanashi, Japan \\ ${ }^{3}$ School of Health Sciences, Shinshu University, Nagano, Japan \\ ${ }^{4}$ Diabetes Center, Kochi Memorial Hospital, Kochi, Japan

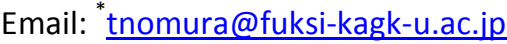

Received 12 August 2014; revised 10 September 2014; accepted 5 October 2014

Copyright (C) 2014 by authors and Scientific Research Publishing Inc.

This work is licensed under the Creative Commons Attribution International License (CC BY). http://creativecommons.org/licenses/by/4.0/

(c) (i) Open Access

\section{Abstract}

Background: The lower extremity muscle strength has been shown to be weaker in patients with diabetes mellitus, primarily due to the effects of diabetic polyneuropathy, a common diabetic complication. With respect to assessments of the lower extremity muscle strength, reference values for the knee extension force in healthy Japanese subjects have been reported. The clinical significance of applying knee extension force in the assessment of efficacy of exercise therapy and patient education has also been demonstrated, and the use of an individualized approach in physical exercise based on the quantitative assessment of the lower extremity muscle strength is becoming one of the most important strategies for promoting effective rehabilitation. Methods: The study is a multicenter, cross-sectional investigation. We will measure the knee extension force and evaluate the diabetic polyneuropathy in patients with type 2 diabetes using uniform equipment and methodology at all centers. We will compare the knee extension force between the subjects with and without diabetic polyneuropathy and will investigate the associations between the knee extension force and both glycemic control and exercise habits. The trial registration is UMIN CTR (UMIN000002810). Discussion: The lower extremity muscle strength has been insufficiently investigated in Japanese patients with diabetes. This study was designed to establish reference values for the knee extension force in Japanese diabetic patients with and without diabetic polyneuropathy.

\footnotetext{
"Corresponding author.
}

How to cite this paper: Nomura, T., Ishiguro, T., Ohira, M., Ikeda, Y. and Watanabe, M. (2014) Multicenter Survey of the Isometric Lower Extremity Strength in Patients with Type 2 Diabetes (MUSCLE-std): Design and Study Protocol. Journal of Diabetes Mellitus, 4, 251-256. http://dx.doi.org/10.4236/jdm.2014.44036 


\section{Keywords}

\section{Muscle Strength, Knee Joint, Diabetes Mellitus, Diabetic Neuropathies, Physical Therapy}

\section{Introduction}

Currently, an estimated 20,500,000 Japanese individuals have diabetes mellitus or are at high risk of developing the disease [1]. Particularly in Japan, diabetes is quickly becoming the disease with the highest incidence [2]. Diabetes commonly accompanies stroke and/or heart disease, afflictions for which patients require rehabilitation [3], and is characterized by the following associated complications that result from chronic hyperglycemia: retinopathy, nephropathy and neuropathy. Diabetic polyneuropathy (DP) is the most frequently occurring complication of diabetes [4], and there is concern that, in the past, most attention had been focused on sensory nerve damage, while less attention had been given to motor nerve damage. One reason for this is thought to be that most clinical determinations of motor nerve damage have been performed [5] using manual muscle testing [6], which requires skill and exhibits a low sensitivity for detecting mild to moderate damage. In recent years, quantitative muscle strength measurements have become widely used, and patients with diabetes have been reported to have impaired lower extremity muscle strength (LMS) levels. In particular, among patients with DP complications, a decline in LMS associated with the severity of DP has been identified [7] [8].

With regard to LMS, reference values for the knee extension force (KEF) obtained from the data of healthy Japanese volunteers have been reported; the values were derived from measurements performed using a handheld dynamometer in conjunction with belt stabilization [9]. The relationships between these values and important everyday movements, including walking and stair climbing have been investigated among elderly patients in foreign countries [10] [11] as well as healthy Japanese subjects [12] [13]. Furthermore, the clinical usefulness of the obtained KEF values for assessing the effectiveness of exercise therapy and patient education has also been reported [14], and the prescription of and guidance in physical exercise based on the quantitative assessment of LMS is becoming one of the most important strategies for promoting effective rehabilitation in Japan.

We previously reported, for the first time, quantitative KEF values in Japanese patients with type 2 diabetes mellitus (T2DM) based on the presence or absence of DP complications [15]. However, due to the small sample size of only 47 patients, the study results were insufficient to establish reference values for KEF in Japanese patients with diabetes. To our knowledge, no other studies have proposed KEF reference values for Japanese patients with diabetes. In this study, we aim to establish KEF reference values for Japanese patients with T2DM based on the relationship with DP.

\section{Methods}

\subsection{Design}

This study is a multicenter, cross-sectional investigation which will be approved by the ethics committee of the Kansai University of Welfare Sciences.

\subsection{Sample Size}

This study is designed to propose KEF reference values for patients with diabetes in relation to sex, age (from 30 to 80 years) and the presence or absence of DP complications. We calculated the required sample size for significance testing regarding the distribution of the measured values and obtained a minimum of 30 subjects with respect to skewness and 50 subjects with respect to kurtosis [16]. The subjects will be divided into 24 groups, and, in order to determine the reference values for each group (e.g., male group, 30's age group and DP complication group), we set the required number of subjects per group at 50 . Therefore, the total number of subjects required is calculated to be 1200 .

Required subjects for this study : 24 groups $\times 50$ subjects $=1200$ subjects 


\subsection{Patients and Practice}

The subjects for this study will be recruited from 30 facilities $^{\mathrm{a}}$ throughout Japan. All selected patients will be diagnosed with T2DM [17]. The inclusion and exclusion criteria were as follows:

\section{Inclusion Criteria}

Inpatients and outpatients with T2DM who are able to provide their written informed consent to participate in the study.

\section{Exclusion Criteria}

1) The inability to adapt to the exercise therapy;

2) No capacity for independent walking;

3) Significant limitations in activities of daily of living;

4) Severe heart and/or respiratory disease;

5) Severe liver dysfunction and/or renal failure (a serum creatinine level over $2.0 \mathrm{mg} / \mathrm{dL}$ );

6) Acute and/or chronic orthopedic disease or presently receiving medical treatment;

7) Non-symmetry of bilateral lower extremity muscle atrophy;

8) Impairment of the lower extremities;

9) Severe infectious disease;

10) Patients requiring surgical treatment;

11) Other conditions resulting in the patient being considered ineligible by the researcher.

\subsection{Measurements}

The date of protocol finalization for this study was October 21, 2009, the start date for patient registration was April 1, 2010 and data collection is planned for five years until March 2015. Regarding the data collection, a procedural manual for measurements has been developed, and all collaborating institutes will use uniform measurements and evaluation methods.

\section{Primary Outcome Measurements}

The measurements of the maximum isometric KEF values (unit: kgf) will be obtained using a hand-held dynamometer with belt stabilization ( $\mu$ Tas M-1 or $\mu$ Tas F-1, Anima Inc., Tokyo, Japan) in the sitting position at $90^{\circ}$ hip joint flexion and $90^{\circ}$ knee joint flexion, similar to the measurement method used in a previous study [9]. The reliability of this measurement method was verified by comparing the muscle strength values obtained using a large isokinetic exercise machine, whose validity has been established [18]. The non-dominant leg (the pivot leg, i.e., the leg one would not kick a ball with) was designated as the leg from which the measurements are to be performed. The KEF (unit: kgf) is divided by the body weight (unit: kg) and expressed as \%KEF (unit: \%) and then converted to Newton units and divided by the moment arm (unit: $\mathrm{m}$ ) to obtain the KEF in N/m units.

\section{Secondary Outcome Measurements}

1) Characteristics: body weight, height, age and the length of the lower leg of the non-dominant leg. The length of the lower leg (moment arm) is to be measured from the knee joint space to the center of the sensor pad of the muscle strength measuring instrument using measuring tape;

2) Diabetes treatment-related information: Duration of diabetes, fasting plasma glucose or postprandial plasma glucose, HbA1c, LDL-cholesterol, HDL-cholesterol, total cholesterol, triglycerides, serum creatinine, fasting insulin, use of diet and/or drug therapy. The duration of diabetes will be determined by a diabetes specialist;

3) Diabetic polyneuropathy assessed according to a simple diagnosis standard neuropathy test [19]: Achilles' tendon reflex, vibratory sense on the medial malleolus, subjective symptoms;

4) Complications associated with diabetes (retinopathy, nephropathy), complications not related to diabetes;

5) Clarify and evaluate the patient's normal exercise habits, drinking habits and smoking habits. The presence of exercise habits is defined as exercising twice a week or more for at least 30 minutes each time. Exercise habits are judged to be in the precontemplation, contemplation, preparation, action, maintenance or termination stage based on the transtheoretical model [20], with subjects in the action stage or later defined as having positive exercise habits.

\subsection{Data Collection}

A dedicated research database will be constructed, and data will be collected at the Kansai University of Welfare Sciences (Figure 1). The database is to be made such that collaborating research centers will be able to verify 


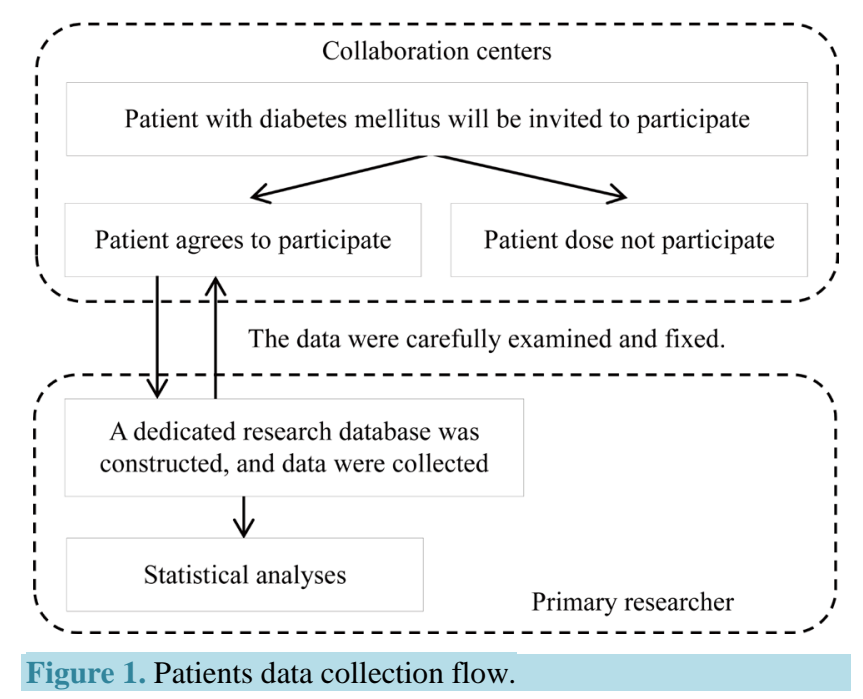

the data from their end. The obtained data will be carefully examined and fixed following verification with the collaborating centers.

\subsection{Statistical Analysis}

All statistical analyses will be conducted using the IBM SPSS Statics 19.0 software program (Chicago, IL, USA). The KEF values will be compared according to sex, age group and presence or absence of DP complications. In addition, the KEF will be analyzed based on the presence or absence of exercise habits, level of diabetes control and complications other than DP, and the association between insulin resistance and the KEF will be assessed [21].

\section{Discussion}

If reference values are not obtained using a measurement method used by health care professionals involved in rehabilitation or diabetes management all over the country, the range of use for the obtained values will obviously be limited. Moreover, even if the versatility is high, the measured values must display a high reliability, equivalent to that of the muscle strength evaluation instruments generally used within the medical field. Accordingly, this study will use a small muscle strength measuring instrument that has been verified to provide muscle strength values comparable to those obtained from isometric exercise machines, for which validity has been established [22]. Because we aimed to establish reference values by using measurements obtained with a small muscle strength measuring instrument that combines reproducibility and reliability, is cheaper than commonly used isometric exercise machines and is progressively being used in clinics involved in rehabilitation, the clinical versatility of the obtained results will be high.

In particular, the results of this study will contribute to standardizing the grading method for determining the severity of DP, effectiveness of exercise therapy and indications for prescribing exercise therapy (such as evaluation criteria for identifying the need for interventions aimed at muscle strengthening, creating designs for developing gradual muscle strengthening methods, estimating the muscle strengthening effects of various physical activities) in diabetic patients. We hope that our results will also be practically applied to related fields, such as sports and health sciences, with the goal to prevent lifestyle diseases and help to determine whether long-term care is required.

\section{Author's Contribution}

T.N. is the primary researcher for this study and both managed the database and drafted the manuscript. T.I. and M.O. are other researchers who participated in the design of the study protocol and helped to draft the manuscript. Y.I. performed the clinical evaluation of the data, participated in determining the study design and coordination and helped to draft the manuscript. M.W. contributed to supplying the fund and helped to draft the manuscript. All authors have read and approved the final manuscript. All authors declare that they have no com- 
peting interests in association with this study.

\section{Endnotes}

\section{0 facilities $^{a}$ : collaboration centers}

Ishikawa Prefectural Central Hospital, Kanazawa-city, Ishikawa; Hidaka Medical Center, Toyooka Public Hospitals' Association, Toyooka-city, Hyogo; Yoka Hospital, Yoka-city, Hyogo; KKR Takamatsu Hospital, Takamatsu-city, Kagawa; Kinashi Obayashi Hospital, Takamatsu-city, Kagawa; Shimanto City Hospital, Shimanto-city, Kochi; Kochi Medical School Hospital, Nankoku-city, Kochi; Chibune General hospital, Osaka-city, Osaka; Aki General Hospital, Aki-city, Kochi; Itami City Hospital, Itami-city, Hyogo; Suita Municipal Hospital, Suita-city, Osaka; Tokyo Medical University Hachioji Medical Center, Hachioji-city, Tokyo; Ishikawa Hospital, Himeji-city, Hyogo; St. Marianna University School of Medicine Hospital, Kawasaki-city, Kanagawa; Kohka Public Hospital, Kohka-city, Shiga; Minami Seikyo Hospital, Nagoya-city, Aichi; Tosei General Hospital, Setocity, Aichi; Osaka Rosai Hospital Research Center for the Promotion of Health and Employment Support, Sakai-city, Osaka; Kasugai Municipal Hospital, Kasugai-city, Aichi; Kanazawa Red Cross Hospital, Kanazawacity, Ishikawa; Hiroshima University Hospital, Hiroshima-city, Hiroshima; Aino Hospital, Ibaraki-city, Osaka; Nagano Chuo Hospital, Nagano-city, Nagano; Kitasato University Hospital, Sagamihara-city, Kanagawa; Kyoto Minami Hospital, Kyoto-city, Kyoto; Steel Memorial Yawata Hospital, Kitakyushu-city, Fukuoka; Okazaki City Hospital, Okazaki-city, Aichi; Saitama Citizens Medical Center, Saitama-city, Saitama; Daiyukai General Hospital, Ichinomiya-city, Aichi; Maruko Central Hospital, Ueda-city, Nagano.

\section{Acknowledgements}

This research was supported by a Grant-in-Aid for Young Scientists (B), Japan Society for the Promotion of Science. In addition, this research was partially supported by a grant from the Kansai University of Welfare Sciences. The authors thank Dr. Misuzu Watanabe for providing advice on the plan for the statistical analysis. The authors also thank the participants and collaborators at the collaboration centers in this study.

\section{References}

[1] Japanese Ministry of Health, Labour and Welfare (2012) National Health and Nutrition Survey Japan, 2012. http://www.mhlw.go.jp/bunya/kenkou/eiyou/h24-houkoku.html

[2] Goto, A., Goto, M., Noda, M. and Tsugane, S. (2013) Incidence of Type 2 Diabetes in Japan: A Systematic Review and Meta-Analysis. PLoS ONE, 8, e74699. http://dx.doi.org/10.1371/journal.pone.0074699

[3] Kohzuki, M. (2010) Diabetes and Disability, the Disabled and Diabetes. Monthly Book Medical Rehabilitation, 117, $1-8$.

[4] Sone, H. (2010) Epidemiology of Diabetic Vascular Complications. The Journal of Japanese College of Angiology, 50, 523-531.

[5] Wikholm, J.B. and Bohannon, R.W. (1991) Hand-Held Dynamometer Measurements: Tester Strength Makes a Difference. Journal of Orthopaedic Sports Physical Therapy, 13, 191-198. http://dx.doi.org/10.2519/jospt.1991.13.4.191

[6] Helen, J.H., Dale, A. and Marybeth, B. (2013) Daniels and Worthingham’s Muscle Testing, Techniques of Manual Examination and Performance Testing. 9th Edition, Saunders, Philadelphia.

[7] Andersen, H., Poulsen, P.L., Mogensen, C.E. and Jakobsen, J. (1996) Isokinetic Muscle Strength in Long-Term IDDM Patients in Relation to Diabetic Complications. Diabetes, 45, 440-445. http://dx.doi.org/10.2337/diab.45.4.440

[8] Andersen, H., Nielsen, S., Mogensen, C.E. and Jakobsen, J. (2004) Muscle Strength in Type 2 Diabetes. Diabetes, 53, 1543-1548. http://dx.doi.org/10.2337/diabetes.53.6.1543

[9] Hirasawa, Y., Hasegawa, T., Matsusita, K. and Yamasaki, Y. (2004) Isometric Knee Extension Strength among Healthy Subjects. Rigakuryohojanaru, 33, 330-333.

[10] Bassey, E.J., Fiatarone, M.A., O’Neill, E.F., Kelly, M., Evans, W.J. and Lipsitz, L.A. (1992) Leg Extensor Power and Functional Performance in Very Old Men and Women. Clinical Science, 82, 321-327.

[11] Rantanen, T. and Avela, J. (1997) Leg Extension Power and Walking Speed in Very Old People Living Independently. The Journals of Gerontology Series A: Biological Sciences and Medical Sciences, 52A, M225-M231. http://dx.doi.org/10.1093/gerona/52A.4.M225

[12] Aoki, U., Yamasaki, H., Yokoyama, H., Omori, Y., Kasahara, M. and Hiraki, K. (2001) The Relationship between 
Stair-Climbing Ability and Knee Extension Strength. Journal of the Japanese Physical Therapy Association, 35, 907910.

[13] Omori, Y., Yokoyama, H., Aoki, U., Kasahara, M., Hiraki, K., Yamasaki, H. and Sasa, M. (2004) Relationship between Isometric Knee Extension Strength and Ability to Rise from a Chair in Elderly Patients. Journal of the Japanese Physical Therapy Association, 31, 106-112.

[14] Yamasaki, H. (2004) Lower Extremities Muscle Strength and Gait Training. Sogo Rihabiriteshon, 32, 813-818.

[15] Nomura, T., Ikeda, Y., Suehiro, T., Nishigami, T., Nakao, S. and Ishida, K. (2006) Relationship between One-Leg Standing Balance and Knee Extension Force in Patients with Type 2 Diabetes. Journal of the Japan Diabetes Society, 49, 227-231.

[16] Ichihara, K. (1990) Statics for Bioscience, Practical Technique and Theory. Nankodo, Tokyo.

[17] The Expert Committee on the Diagnosis and Classification of Diabetes Mellitus (1997) Report of the Expert Committee on the Diagnosis and Classification of Diabetes Mellitus. Diabetes Care, 20, 1183-1197.

[18] Hirasawa, Y., Hasegawa, T., Sasa, M. and Yamasak, H. (2005) The Validity of the Isometric Knee Extension Muscle Strength by Hand Held Dynamometer. Sogo Rihabiriteshon, 33, 375-377.

[19] The Japan Diabetes Society (2013) Evidence-Based Practice Guideline for the Treatment of Diabetes in JAPAN 2013. Nankodo, Japan.

[20] Burbank, P.M. and Riebe, D. (2001) Promoting Exercise and Behavior Change in Older Adults. Springer, Berlin.

[21] Nomura, T., Ikeda, Y., Nakao, S., Ito, K., Ishida, K., Suehiro, T. and Hashimoto, K. (2007) Muscle Strength Is a Marker of Insulin Resistance in Patients with Type 2 Diabetes: A Pilot Study. Endocrine Journal, 54, 791-796. http://dx.doi.org/10.1507/endocrj.K07-055

[22] Yamasaki, H. (2007) Muscle Strength Measurements According to Instrument. Sogo Rihabiriteshon, 35, 724-725.

\author{
Abbreviations \\ DN: Diabetic Neuropathy; \\ DP: Diabetic Polyneuropathy; \\ KEF: Knee Extension Force; \\ LMS: Lower Extremity Muscle Strength; \\ T2DM: Type 2 Diabetes Mellitus.
}


Scientific Research Publishing (SCIRP) is one of the largest Open Access journal publishers. It is currently publishing more than 200 open access, online, peer-reviewed journals covering a wide range of academic disciplines. SCIRP serves the worldwide academic communities and contributes to the progress and application of science with its publication.

Other selected journals from SCIRP are listed as below. Submit your manuscript to us via either submit@scirp.org or Online Submission Portal.
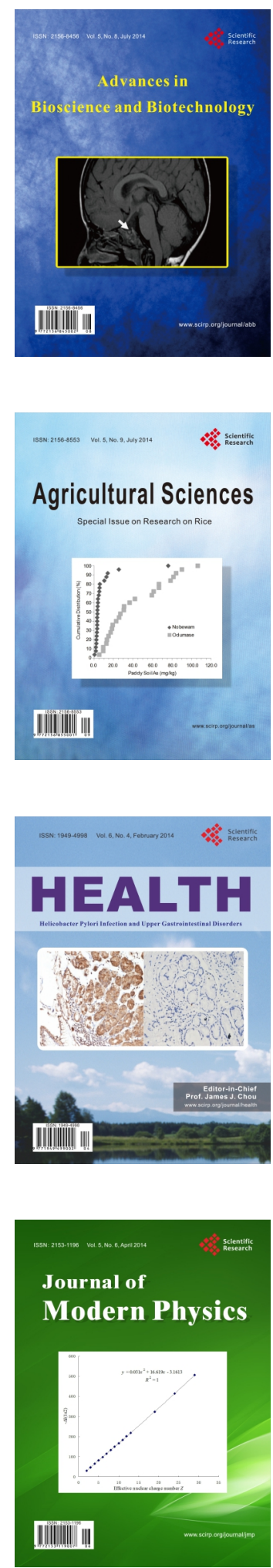
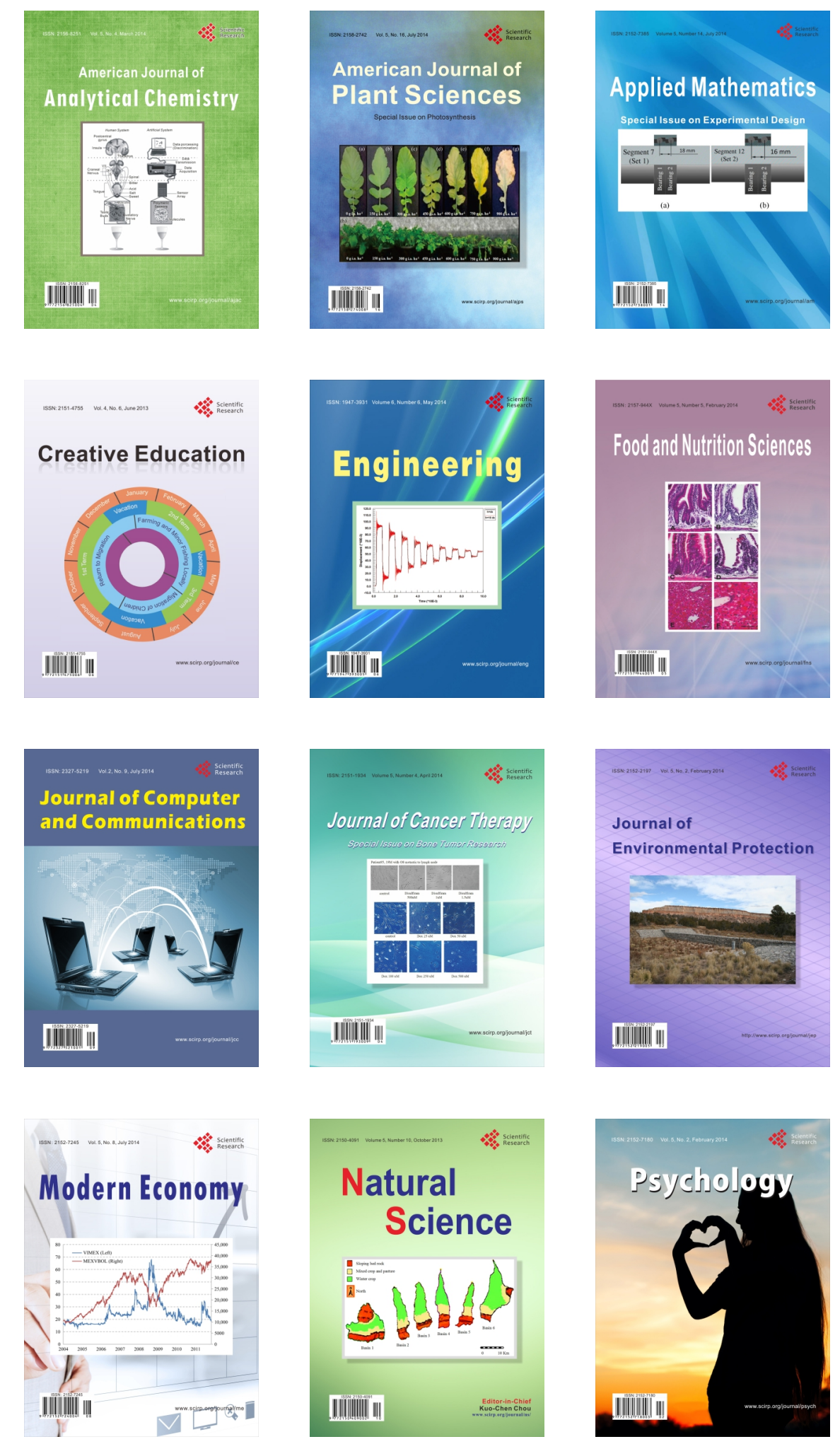